\title{
ASSOCIATION OF INTERLEUKIN-10 -592A >C AND -819T>C POLYMORPHISMS WITH GASTRIC CANCER RISK: A SYSTEMATIC REVIEW AND META-ANALYSIS OF 44 CASE-CONTROL STUDIES
}

\author{
Associação de polimorfismos da Interleucina-10 -592A >C e -819T>C com risco de câncer gástrico: Revisão sistemática e \\ metanálise de 44 estudos de caso-controle

\begin{abstract}
Mansour MOGHMI ${ }^{1}$, Amir ARJMANDI ${ }^{2}$, Kazem AGHILI ${ }^{3}$, Mohammadali JAFARI ${ }^{4}$, Masoud ZARE-SHEHNEH ${ }^{2}$ Shohreh RASTEGAR ${ }^{5}$, Seyed Mojtaba ABOLBAGHAEl ${ }^{6}$, Hossein NEAMATZADEH ${ }^{2}$
\end{abstract}

\begin{abstract}
How to cite this article: Moghmi M, Arjmandi A, Aghili K, Jafari M, Zare-Shehneh M, Rastegar S, Abolbaghaei SM, Neamatzadeh H. Associação de polimorfismos da Interleucina-10 -592a>c e -819t>c com risco de câncer gástrico: revisão sistemática e metanálise de 44 estudos de caso-controle. ABCD Arq Bras Cir Dig. 2019;32(1):e1415. DOI: /10.1590/0102-672020180001e1415
\end{abstract}

From the ${ }^{1}$ Shahid Sadoughi University of Medical Sciences, Pathology, Yazd, Yazd; ${ }^{2}$ Shahid Sadoughi University of Medical Sciences, Medical Genetics, Yazd, Yazd; ${ }^{3}$ Shahid Sadoughi University of Medical Sciences, Radiology, Yazd, Yazd; ${ }^{4}$ Shahid Sadoughi University of Medical Sciences, Emergency Medicine, Yazd, Yazd; ${ }^{5}$ Shahid Sadoughi University of Medical Sciences, Anesthesiology, Yazd, Yazd; ${ }^{6}$ Shahid Beheshti University of Medical Sciences, Forensic Medicine, Tehran, Tehran, Iran.

HEADINGS - Stomach neoplasms Interleukin-10. Polymorphism, genetic. Meta-analysis
ABSTRACT - Introduction: A series of studies have evaluated the association between $-592 \mathrm{~A}>\mathrm{C}$ and $-819 \mathrm{~T}>\mathrm{C}$ polymorphisms in the promoter regions of Interleukin-10 (IL-10) and gastric cancer (GC) risk. However, the results remain inconclusive. Objective: To better understand the association of the polymorphisms with GC risk, we performed a comprehensive meta-analysis. Method: An electronic search was performed of several databases to identify relevant studies up to April 2018. Results: A total of 44 case-control studies, including 26 studies on IL-10 $-592 \mathrm{~A}>\mathrm{C}(5,332$ cases and 8,272 controls) and 18 studies on IL-10 -819T>C (3,431 cases and 6,109 controls) were selected. Overall, $-592 \mathrm{~A}>\mathrm{C}$ polymorphism was associated with the risk of $\mathrm{GC}$ under the heterozygote model $(\mathrm{OR}=1.153,95 \% \mathrm{Cl}=1.020-1.305, \mathrm{p}=0.023)$, but not $-819 \mathrm{~T}>\mathrm{C}$ polymorphism. When stratified by ethnicity, significant association was only observed in the Asians under the allele model $(\mathrm{OR}=1.153,95 \% \mathrm{Cl}=1.007-1.320, \mathrm{p}=0.040)$ and the heterozygote model $(\mathrm{OR}=1.218,95 \% \mathrm{Cl}=1.076-1.379, \mathrm{p}=0.002)$ for $-592 \mathrm{~A}>\mathrm{C}$. Conclusion: The current metaanalysis results inconsistent with previous meta-analyses; showed that the IL-10 -592A $>C$ polymorphism, but not $-819 \mathrm{~T}>\mathrm{C}$ polymorphism, may be contributed to the susceptibility of GC in overall and Asian populations.

\section{Correspondence:}

Amir Arjmandi

E-mail: hn 1364@yahoo.com;

hn_1364@yahoo.com

Financial source: none

Conflict of interest: none

Received for publication: 21/08/2018 Accepted for publication: $11 / 10 / 2018$

DESCRITORES - Neoplasias Gástricas Interleucina-10. Polimorfismo genético. Metanálise
RESUMO - Introdução: Uma série de estudos avaliou a associação entre os polimorfismos $-592 \mathrm{~A}>\mathrm{C}$ e -819T>C nas regiões promotoras do risco de interleucina-10 (IL-10) e câncer gástrico (GC). No entanto, os resultados permanecem inconclusivos. Objetivo: Para entender melhor a associação dos polimorfismos com o risco de GC, realizamos uma meta-análise abrangente. Método: Foi realizada busca eletrônica de vários bancos de dados para identificar estudos relevantes até abril de 2018. Resultados: Um total de 44 estudos caso-controle, incluindo 26 estudos sobre IL-10 -592A >C (5.332 casos e 8.272 controles) e 18 estudos sobre IL-10 -819T >C (3.431 casos e 6.109 controles) foram selecionados. No geral, o polimorfismo $-592 \mathrm{~A}>\mathrm{C}$ foi associado ao risco de GC sob o modelo heterozigoto $(\mathrm{OR}=1,153,95 \% \mathrm{IC}=1,020$ $1,305, p=0,023)$, mas não polimorfismo $-819 T>C$. Quando estratificada por etnia, associação significativa foi observada apenas nos asiáticos sob o modelo alelo $(O R=1,153$, IC 95\%=1,007$1,320, p=0,040)$ e o modelo heterozigoto $(O R=1,218$, IC $95 \%=1,076-1,379, p=0,002)$ para -592A>C. Conclusão: Os atuais resultados são inconsistentes com metanálises anteriores; mostrou que o polimorfismo IL-10 $-592 \mathrm{~A}>\mathrm{C}$, mas não o polimorfismo $-819 \mathrm{~T}>\mathrm{C}$, pode ter contribuído para a suscetibilidade de GC em populações globais e asiáticas. (cc) BY This is an open-access article distributed under the terms of the Creative Commons Attribution License.

\section{INTRODUCTION}

G astric cancer (GC) is the $5^{\text {th }}$ most common cancer and second leading cause of cancer-related deaths globally ${ }^{21,32,36}$. In 2016 there were an estimated 26,370 new cases of GC in the United States ${ }^{39}$. The recent years have brought much progress regarding the genetics of GC and the number of confirmed GC associated SNPs and genes have risen dramatically ${ }^{4,48}$. In addition, several studies have supported the concept that environmental factors are critical components of GC pathogenesis ${ }^{52}$. However, genetic factors may modify the propensity for GC development through an alteration of the inflammatory state and may also interact with other risk factors ${ }^{44}$.

Presently the mechanisms of the etiology and progression of GC are far from clear ${ }^{4,38}$. Several genes have been identified to be associated with GC risk, including Interleukin-10 (IL-10). IL-10 is a multifunctional cytokine with anti-inflammatory properties, which has been reported involving in the some malignancies progress 
and development ${ }^{22,48}$. The human gene that encodes IL-10 (Gene ID: 3586) maps to the long arm of chromosome 1 (1q31-32), which contains three most common 21082A>G (rs1800896), 2592C > A (rs1800872) and 2829C > T (rs1800871) polymorphisms located within the promoter region. These polymorphisms are associated with low/high amount of IL-10 secretion ${ }^{31,45}$.

A series of epidemiological studies have reported the association of $-592 \mathrm{~A}>\mathrm{C}(\mathrm{rs} 1800872)$ and $-819 \mathrm{~T}>\mathrm{C}(\mathrm{rs} 3021097)$ polymorphisms of IL-10 gene with GC risk $22,31,45$, but the results remain conflicting rather than conclusive. Some meta-analyses previously published regarding the association of $-592 \mathrm{~A}>\mathrm{C}$ (rs1800872) and -819T>C (rs3021097) polymorphisms with $\mathrm{GC}$ risk $4,48,55$. A few studies were not included in these metaanalyses and also original studies with larger sample sizes in different ethnicity have been published since then. In addition, some of the previous meta-analyses have reported conflicting conclusions.

Hence, we performed this meta-analysis to evaluate whether the IL-10-592A >C and -819T >C polymorphisms contributed to the susceptibility of GC. Based on our knowledge, this is the most comprehensive and accurate meta-analysis of the association of IL-10 -592A >C and -819T >C polymorphisms with $\mathrm{GC}$ risk.

METHOD

\section{Search strategy}

The electronic databases of the US National Library of Medicine's PubMed, EMBASE, Web of Knowledge, Google Scholar, Wanfang, Chinese National Knowledge Infrastructure $(\mathrm{CNKI})$, and Chinese Biomedical Literature Database (CBM) were systematically searched to retrieve potential publications that assessed the association between $-592 \mathrm{~A}>\mathrm{C}$ and $-819 \mathrm{~T}>\mathrm{C}$ polymorphisms of IL-10 gene and GC risk up to April 10, 2018. Key search terms used were as follows: (gastric cancer OR gastric neoplasm OR stomach neoplasms) AND (Interleukin-10 OR IL-10) AND (-592A > C OR rs1800872) AND (-819T>C OR rs 1800871) AND (Polymorphism OR SNP OR single nucleotide polymorphism OR variation OR mutation). This meta-analysis included only publications relating to humans, covering all relevant written in English and Chinese publications with available full-text articles. Reference lists of retrieved articles, review articles, and previous meta-analysis were also manually searched to avoid missing relevant studies.

\section{Inclusion and exclusion criteria}

Studies were included in the meta-analysis if they met the following criteria: 1) full text available; 2 ) case-control or cohort studies; 3 ) studies focus on the association of $-592 \mathrm{~A}>\mathrm{C}$ (rs1800872) and -819T > C (rs3021097) polymorphisms of IL-10 gene with GC risk; 4) sufficient published data for genotype and allele frequencies to calculate the Odds Ratio (OR) and 95\% confidence interval $(\mathrm{Cl})$.

Major reasons for exclusion of studies were as follows: 1) abstract, review articles, case reports, unpublished data and comments; 2) studies with overlapped or duplicate data; 3 ) no healthy control group established in the study; 4) studies with unclear or ambiguous data or genotype frequencies. When duplicated studies were published by the same author obtained from the same patient sample, only the one with the largest sample size was included in this meta-analysis.

\section{Data extraction}

Data were carefully extracted from all eligible studies independently by two investigators according to the inclusion and exclusion criteria. The following data were collected from each study: first author, year of publication, country origin, ethnicity, total number of cases and controls, the frequencies of genotypes, minor allele frequencies (MAFs), p-value for Hardy-Weinberg equilibrium (HWE). In case of disagreement (in the data extraction), consensus was resolved through consensus, or a third author would assess these articles. In the current meta-analysis, the quality of selected studies was tested by the confirmation of HWE in control groups, and studies without the confirmation of HWE in controls were defined as low-quality studies, while studies with the confirmation of HWE in controls were defined as high-quality studies (Table 1).

\section{Statistical analysis}

All meta-analyses were conducted using Comprehensive Meta-Analysis (CMA) software (USA, version 2.2.064) and a $p$ value below 0.05 was considered statistically significant. The strength of the association of $-592 \mathrm{~A}>\mathrm{C}$ and $-819 \mathrm{~T}>\mathrm{C}$ polymorphisms of IL-10 gene with GC risk was estimated by crude odds ratios (ORs) with corresponding 95\% confidence intervals (Cls). The significance of the pooled OR was determined by the Z-test. An allele contrast model (C vs. T), homozygote model (CC vs. TT), heterozygote model (CT vs. TT), dominant $(C C+C T$ vs. TT), and recessive (CC vs. CT+TT) model were used for IL-10-819T>C polymorphism. An allele contrast model (C vs. A), homozygote model (CC vs. AA), heterozygote model ( $C A$ vs. $A A)$, dominant $(C C+C A$ vs. $A A)$, and recessive (CC vs. $(A+A A)$ model were used for $-592 A>C$ polymorphism. The Cochran chi-square-based Q statistical test was used to evaluate statistical between-study heterogeneity (with $p<0.05$ for statistical significance). In addition, a quantitative measure of between-study heterogeneity was also investigated using the $I^{2}$ statistic, and which the between-study heterogeneity was considered low, moderate, and high based on $\mathrm{I}^{2}$ values of $25 \%, 50 \%$, and $75 \%$, respectively ${ }^{18}$. If the between-study heterogeneity was statistically significant the random effects model ${ }^{7}$ was applied; otherwise, the fixed effects model 29 was used. The sensitivity analysis was performed to assess the contribution of individual studies to pooled effect estimate by sequentially removing each study one at a time and computing differential estimates for rest. In addition, sensitivity analysis was performed by excluding the low quality studies to test the stability of the results. Publication bias was examined using the Begg's funnel plot and Egger's test ${ }^{2,9}$. If publication bias existed, the Duval and Tweedie non-parametric "trim and fill" method was used to adjust for it. Subgroup analyses by ethnicity and studies quality (by HWE status) were performed subsequently. The distribution of genotypes in control groups was evaluated for a departure from HWE using chi-square test.

\section{RESULTS}

\section{Characteristics of studies}

Tables 1 and 2 showed the characteristics of all the eligible studies selected in the meta-analysis. The study selection processes were presented in Figure 1 (PRISMA 2009 Flow Diagram). We evaluated all the retrieved studies by examining titles, abstracts and conclusions. According to the criteria eligibility, 44 studies in 29 publications was identified regarding the association between the IL-10 -592A >C and -819T>C polymorphisms with susceptibility to the GC. All of these 44 case-control studies provided sufficient data to calculate the association between

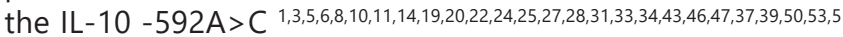
${ }^{4}$ and $-819 T>C 1,5,14,19,22-24,26,27,33,37,43,42,46,47,51,53,54$ polymorphisms with risk of GC. The characteristics of the selected studies are summarized in Tables 1 and 3. Among these studies, 26 case-control studies evaluated the association of the $-592 \mathrm{~A}>\mathrm{C}$ polymorphism with GC with 5,332 cases and 8,272 controls, 
included five groups of Caucasians 5,10,11,19,53, 16 groups of Asians $8,14,20,22,24,25,27,28,33,34,37,43,47,46,50,54$, and five Latinos populations $1,3,6,31,39$ (Table 1). While, 18 case-control studies evaluated the association between the $-819 \mathrm{~T}>\mathrm{C}$ polymorphisms and GC risk, with 3,431 cases and 6,109 controls, included three groups of Caucasians 5,19,53, 14 groups of Asians 14,22-24,26,27,33,37,42,43,46,47,51,54 and one Latinos populations ${ }^{1}$ (Table 2). The countries of these studies included China, Korea, Japan, India, USA, Italy, Finland, Spain, Netherland, Costa Rica, Brazil, Mexico and Chile. All the genotype distributions of controls were in agreement with HWE for IL-10 -592A >C and -819T >C polymorphisms except for nine studies in five publications $11,21,30,31,40$. Therefore, 35 of 44 case-control studies were defined as high-quality studies (Tables 1 and 2).

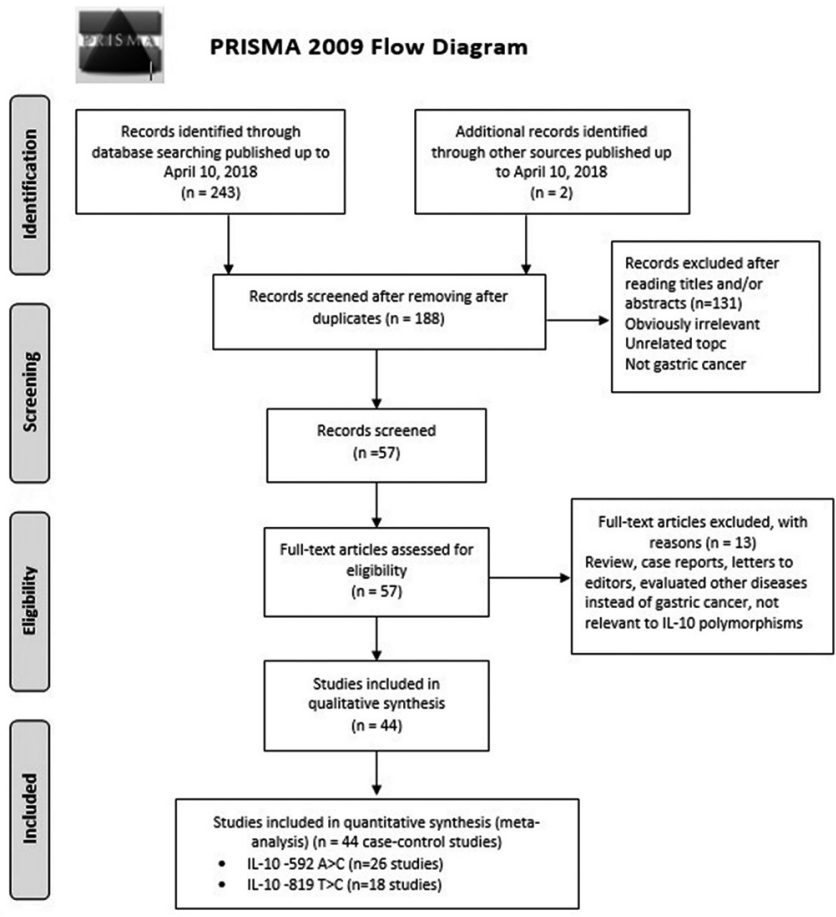

FIGURE 1 - Flow chart of studies selection in this meta-analysis (IL-10 -592A >C and -819T>C Polymorphisms)

\section{Meta-analysis}

IL-10 -592A >C Polymorphism

Table 3 listed the main results of the meta-analysis of IL-10 -592A >C polymorphism and GC risk. When all the eligible studies were pooled into the meta-analysis of IL-10 $-592 \mathrm{~A}>\mathrm{C}$ polymorphism, a significant association was found only under the heterozygote model (CA vs. AA: OR $=1.153,95 \%$ $\mathrm{Cl}=1.020-1.305, \mathrm{p}=0.023$, Figure $2 \mathrm{~A}$ ). In addition, significant between-study heterogeneity was detected in all genetic models. When stratified by ethnicity, a significant association between of IL-10 -592A >C polymorphism and increased GC risk among Asians was detected under the allele model ( $C$ vs. $A: O R=1.153,95 \% C l=1.007-1.320, p=0.040$ ) and the heterozygote model (CA vs. AA: OR=1.218, 95\% Cl=1.076$1.379, p=0.002$ ), but not among Caucasian and Latinos populations. Subgroup analysis of studies with high quality showed that there was a significant association between IL-10 -1082 A>G polymorphism and increased risk of GC only under the allele model $(\mathrm{OR}=1.154,95 \% \mathrm{Cl}=1.004-1.326$, $\mathrm{p}=0.044$, Table 2).

\section{IL-10 -819T>C Polymorphism}

Table 4 and Figure $2 \mathrm{~B}$ showed the main results of the meta-analysis of IL-10 -819T >C polymorphism and GC risk. When all the eligible studies were pooled into the metaanalysis of IL-10 -819T >C polymorphism, no significant association was observed in any genetic model. In the stratified analyses based on ethnicity and studies quality, there was not still significant association between IL-10 -819T > C polymorphism and risk of GC.

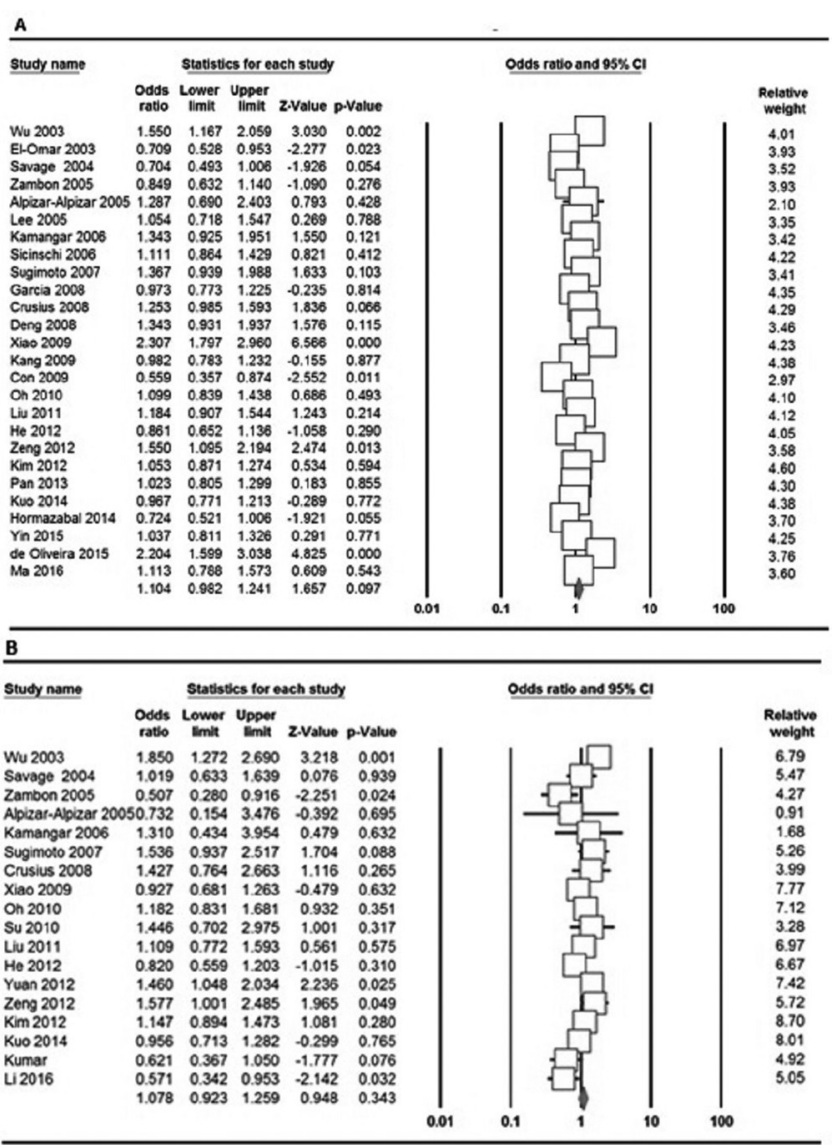

FIGURE 2 - Forest plot of the association of IL-10 $-592 \mathrm{~A}>\mathrm{C}$ and -819 T > C Polymorphisms with GC: A) $-592 \mathrm{~A}>\mathrm{C}$ (homozygote model: C vs. A); B) -819T>C (dominant model: $\mathrm{CC}+\mathrm{CT}$ vs. TT)

Heterogeneity and sensitivity analysis

As shown in Tables 3 and 4, there was a significant betweenstudy heterogeneity for IL-10 $-592 \mathrm{~A}>\mathrm{C}$ polymorphism under all genetic models (C vs. $A: P h=0.001 ; C C$ vs. $A A: P h=0.001$; CA vs. $A A: P h=0.009 ; C C+C A$ vs. $A A: P h=0.001 ; C C$ vs. $C A+A A:$ $\mathrm{Ph}=0.001)$, and for of IL-10 -819T $>C$ (rs3021097) polymorphism under four genetic models ( $C$ vs. $A: P h=0.001 ; C C$ vs. TT: $\mathrm{Ph}=0.0160 .001 ; \mathrm{CT}$ vs. TT: $\mathrm{Ph}=021 ;$ and $C C+C T$ vs. TT: $\mathrm{Ph}=0.002)$, except the recessive genetic model (CC vs. $C T+T T: P h=0.150)$. We performed sensitivity analysis by omitting one study at a time and calculating the pooled ORs again. However, the results did not show any significant statistical differences when studies were omitted. Therefore, the stability of the study was not influenced by any individual study.

\section{Publication bias}

Both Begg's funnel plot and Egger's test were carried out to evaluate the publication bias of the studies. Tables 3 and 4 presents the results of Begg's funnel plot and Egger's test under the five genetic models. As shown in Figure 3A, the shapes of the Begg's funnel plots under the allele model of IL-10 -592A >C polymorphism shown approximately symmetrical and significant evidence of publication bias was not observed by the Egger's test. As for the IL-10 -819T>C polymorphism, the shapes of the Begg's funnel plots under the heterozygote comparison model seemed symmetrical (Figure 3B). In addition, the Egger's tests (all $p$ values for Egger's test $>0.05$ ) also showed that there was no evidence of publication bias for both polymorphisms. 
TABLE 1 -The general characteristics of eligible studies in the meta-analysis of IL-10 -592A>C

\begin{tabular}{|c|c|c|c|c|c|c|c|c|c|c|c|c|c|c|c|}
\hline \multirow{3}{*}{ First Author } & \multirow{3}{*}{$\begin{array}{l}\text { Country } \\
\text { (Ethnicity) }\end{array}$} & \multirow{3}{*}{ Case } & \multirow{3}{*}{ Control } & \multicolumn{5}{|c|}{ Cases } & \multicolumn{5}{|c|}{ Controls } & \multirow{3}{*}{ MAFs } & \multirow{3}{*}{ HWE } \\
\hline & & & & \multicolumn{3}{|c|}{ Genotypes } & \multicolumn{2}{|c|}{ Allele } & \multicolumn{3}{|c|}{ Genotypes } & \multicolumn{2}{|c|}{ Allele } & & \\
\hline & & & & AA & CA & $\mathrm{CC}$ & A & C & AA & CA & CC & A & C & & \\
\hline Wu $2003^{46}$ & China (Asian) & 220 & 230 & 88 & 105 & 27 & 281 & 159 & 127 & 83 & 20 & 337 & 123 & 0.267 & 0.231 \\
\hline El-Omar $2003^{10}$ & USA(Caucasian) & 314 & 210 & 35 & 101 & 178 & 171 & 457 & 13 & 70 & 127 & 96 & 324 & 0.771 & $=0.001$ \\
\hline Savage $2004^{37}$ & China (Asian) & 84 & 386 & 9 & 39 & 36 & 57 & 111 & 49 & 166 & 171 & 205 & 567 & 0.734 & 0.382 \\
\hline Zambon $2005^{53}$ & Italy (Caucasian) & 129 & 644 & 17 & 42 & 70 & 76 & 182 & 46 & 245 & 353 & 337 & 951 & 0.738 & 0.696 \\
\hline $\begin{array}{l}\text { Alpizar-Alpizar } \\
2005^{1}\end{array}$ & Costa Rica(Latinos) & 45 & 45 & 3 & 20 & 21 & 27 & 63 & 5 & 21 & 18 & 32 & 58 & 0.647 & 0.761 \\
\hline Lee $2005^{25}$ & Korea (Asian) & 122 & 120 & 52 & 62 & 8 & 166 & 78 & 53 & 60 & 7 & 166 & 74 & 0.308 & 0.059 \\
\hline Kamangar $2006^{19}$ & $\begin{array}{l}\text { Finland } \\
\text { (Caucasian) }\end{array}$ & 112 & 237 & 6 & 38 & 68 & 50 & 174 & 17 & 82 & 109 & 132 & 342 & 0.721 & 0.775 \\
\hline Sicinschi $2006^{39}$ & Mexico (Latinos) & 181 & 369 & 40 & 90 & 51 & 170 & 192 & 95 & 176 & 98 & 366 & 372 & 0.504 & 0.376 \\
\hline Sugimoto $2007{ }^{43}$ & Japan (Asian) & 105 & 168 & 43 & 54 & 8 & 140 & 70 & 88 & 70 & 10 & 246 & 90 & 0.267 & 0.419 \\
\hline Garcia $2008^{11}$ & Spain (Caucasian) & 404 & 404 & 24 & 143 & 237 & 191 & 617 & 28 & 131 & 245 & 187 & 621 & 0.768 & 0.075 \\
\hline Crusius $2008^{5}$ & $\begin{array}{l}\text { Netherland } \\
\text { (Caucasian) }\end{array}$ & 237 & 1122 & 11 & 78 & 148 & 100 & 374 & 83 & 397 & 642 & 563 & 1681 & 0.749 & 0.049 \\
\hline Deng $2008^{8}$ & China (Asian) & 125 & 110 & 30 & 39 & 56 & 99 & 151 & 39 & 25 & 46 & 103 & 117 & 0.531 & $=0.001$ \\
\hline Xiao 200947 & China (Asian) & 220 & 624 & 100 & 100 & 20 & 300 & 140 & 272 & 283 & 69 & 1038 & 210 & 0.337 & 0.718 \\
\hline Kang 200920 & Korea (Asian) & 333 & 332 & 142 & 157 & 34 & 441 & 225 & 146 & 145 & 41 & 437 & 227 & 0.341 & 0.591 \\
\hline Con $2009^{3}$ & Costa Rica(Latinos) & 52 & 191 & 10 & 26 & 16 & 44 & 60 & 23 & 65 & 103 & 111 & 271 & 0.709 & 0.015 \\
\hline Oh $2010^{33}$ & China (Asian) & 178 & 362 & 77 & 81 & 20 & 235 & 121 & 167 & 159 & 36 & 493 & 231 & 0.319 & 0.861 \\
\hline Liu $2011^{27}$ & China (Asian) & 234 & 243 & 99 & 96 & 39 & 294 & 174 & 109 & 106 & 28 & 324 & 162 & 0.333 & 0.772 \\
\hline He $2012{ }^{14}$ & China (Asian) & 196 & 248 & 82 & 96 & 18 & 260 & 132 & 92 & 128 & 28 & 312 & 184 & 0.371 & 0.095 \\
\hline Zeng $2012^{54}$ & China (Asian) & 151 & 153 & 59 & 77 & 15 & 195 & 107 & 80 & 66 & 7 & 226 & 80 & 0.261 & 0.147 \\
\hline Kim $2012{ }^{22}$ & Korea (Asian) & 495 & 495 & 231 & 214 & 50 & 676 & 314 & 248 & 191 & 56 & 687 & 303 & 0.306 & 0.041 \\
\hline Pan $2013^{34}$ & China (Asian) & 308 & 308 & 144 & 128 & 36 & 416 & 200 & 142 & 135 & 31 & 419 & 197 & 0.319 & 0.895 \\
\hline Kuo $2014{ }^{24}$ & China (Asian) & 358 & 358 & 186 & 134 & 38 & 506 & 210 & 358 & 180 & 141 & 501 & 215 & 0.340 & $=0.001$ \\
\hline $\begin{array}{l}\text { Hormazabal } \\
2014^{31}\end{array}$ & Chile (Latinos) & 147 & 172 & 19 & 73 & 55 & 111 & 183 & 11 & 83 & 78 & 105 & 239 & 0.694 & 0.070 \\
\hline Yin $2015^{50}$ & China (Asian) & 228 & 461 & 112 & 96 & 20 & 320 & 136 & 235 & 184 & 42 & 654 & 268 & 0.290 & 0.490 \\
\hline de Oliveira $2015^{6}$ & Brazil (Latinos) & 207 & 240 & 104 & 82 & 21 & 290 & 124 & 169 & 64 & 7 & 402 & 78 & 0.162 & 0.753 \\
\hline Ma $2016^{28}$ & China (Asian) & 147 & 150 & 67 & 63 & 17 & 197 & 97 & 71 & 67 & 12 & 208 & 92 & 0.303 & 0.486 \\
\hline
\end{tabular}

TABLE 2 - The general characteristics of eligible studies in the meta-analysis of IL-10 -819T>C

\begin{tabular}{|c|c|c|c|c|c|c|c|c|c|c|c|c|c|c|c|}
\hline \multirow{3}{*}{ First Author } & \multirow{3}{*}{$\begin{array}{l}\text { Country } \\
\text { (Ethnicity) }\end{array}$} & \multirow{3}{*}{ Case } & \multirow{3}{*}{ Control } & \multicolumn{5}{|c|}{ Cases } & \multicolumn{5}{|c|}{ Controls } & \multirow{3}{*}{ MAFs } & \multirow{3}{*}{ HWE } \\
\hline & & & & \multicolumn{3}{|c|}{ Genotypes } & \multicolumn{2}{|c|}{ Allele } & \multicolumn{3}{|c|}{ Genotypes } & \multicolumn{2}{|c|}{ Allele } & & \\
\hline & & & & TT & CT & $\mathrm{CC}$ & $\mathrm{T}$ & C & TT & $\mathrm{CT}$ & $\mathrm{CC}$ & $\mathrm{T}$ & C & & \\
\hline Wu $2003^{46}$ & China (Asian) & 220 & 230 & 88 & 105 & 27 & 281 & 159 & 127 & 83 & 20 & 337 & 123 & 0.267 & 0.231 \\
\hline Savage $2004^{37}$ & China (Asian) & 84 & 382 & 37 & 38 & 9 & 112 & 56 & 170 & 163 & 49 & 503 & 261 & 0.341 & 0.314 \\
\hline Zambon $2005^{53}$ & Italy (Caucasian) & 129 & 644 & 17 & 42 & 70 & 76 & 182 & 46 & 245 & 353 & 337 & 951 & 0.738 & 0.696 \\
\hline $\begin{array}{l}\text { Alpizar-Alpizar } \\
2005^{1}\end{array}$ & $\begin{array}{c}\text { Costa Rica } \\
\text { (Latinos) }\end{array}$ & 45 & 45 & 4 & 16 & 25 & 24 & 66 & 3 & 24 & 18 & 30 & 60 & 0.666 & 0.179 \\
\hline Kamangar $2006^{19}$ & $\begin{array}{c}\text { Finland } \\
\text { (Caucasian) }\end{array}$ & 98 & 152 & 5 & 35 & 58 & 45 & 151 & 10 & 62 & 80 & 114 & 222 & 0.730 & 0.662 \\
\hline Sugimoto $2007{ }^{43}$ & Japan (Asian) & 105 & 168 & 42 & 57 & 6 & 141 & 69 & 86 & 73 & 9 & 245 & 91 & 0.270 & 0.194 \\
\hline Crusius $2008^{5}$ & $\begin{array}{l}\text { European } \\
\text { (Caucasian) }\end{array}$ & 229 & 1094 & 12 & 72 & 145 & 96 & 362 & 80 & 378 & 636 & 538 & 1650 & 0.754 & 0.023 \\
\hline Xiao 200947 & China (Asian) & 220 & 624 & 100 & 100 & 20 & 300 & 140 & 272 & 283 & 69 & 827 & 421 & 0.337 & 0.718 \\
\hline Oh $2010^{33}$ & China (Asian) & 188 & 379 & 81 & 87 & 20 & 249 & 127 & 179 & 158 & 42 & 516 & 242 & 0.319 & 0.425 \\
\hline Su $2010^{42}$ & China (Asian) & 43 & 100 & 18 & 21 & 4 & 57 & 29 & 51 & 43 & 6 & 145 & 55 & 0.275 & 0.433 \\
\hline Liu $2011^{27}$ & China (Asian) & 234 & 243 & 99 & 96 & 39 & 294 & 174 & 109 & 106 & 28 & 324 & 162 & 0.333 & 0.772 \\
\hline He $2012^{14}$ & China (Asian) & 196 & 248 & 82 & 96 & 18 & 260 & 132 & 92 & 128 & 28 & 312 & 184 & 0.371 & 0.095 \\
\hline Yuan $2012^{51}$ & China (Asian) & 279 & 296 & 108 & 129 & 42 & 345 & 213 & 142 & 120 & 34 & 404 & 188 & 0.317 & 0.265 \\
\hline Zeng $2012^{54}$ & China (Asian) & 151 & 153 & 60 & 80 & 11 & 200 & 102 & 78 & 65 & 10 & 221 & 85 & 0.277 & 0.466 \\
\hline Kim $2012{ }^{22}$ & Korea (Asian) & 495 & 495 & 231 & 214 & 50 & 676 & 314 & 248 & 191 & 56 & 687 & 303 & 0.306 & 0.041 \\
\hline Kuo $2014{ }^{24}$ & China (Asian) & 358 & 358 & 190 & 132 & 36 & 512 & 204 & 186 & 132 & 40 & 504 & 212 & 0.296 & 0.028 \\
\hline Kumar $2015^{23}$ & India (Asian) & 200 & 250 & 36 & 103 & 61 & 175 & 225 & 30 & 119 & 101 & 179 & 321 & 0.642 & 0.574 \\
\hline Li 201626 & China (Asian) & 157 & 248 & 36 & 83 & 38 & 155 & 159 & 36 & 127 & 85 & 199 & 297 & 0.598 & 0.300 \\
\hline
\end{tabular}


TABLE 3 -The meta-analysis of IL-10 -592A > C polymorphism and risk of GC

\begin{tabular}{|c|c|c|c|c|c|c|c|c|c|c|c|}
\hline \multirow{2}{*}{ Subgroup } & \multirow{2}{*}{$\begin{array}{c}\text { Study } \\
\text { number }\end{array}$} & \multirow{2}{*}{ Genetic model } & \multirow{2}{*}{$\begin{array}{l}\text { Type of } \\
\text { model }\end{array}$} & \multicolumn{2}{|c|}{ Heterogeneity } & \multicolumn{4}{|c|}{ Odds ratio } & \multicolumn{2}{|c|}{ Publication Bias } \\
\hline & & & & $12(\%)$ & $\mathrm{PH}$ & OR & $95 \% \mathrm{Cl}$ & Ztest & POR & PBeggs & PEggers \\
\hline \multirow[t]{5}{*}{ Overall } & 26 & C vs. A & Random & 76.40 & $=0.001$ & 1.104 & $0.982-1.241$ & 1.657 & 0.097 & 0.724 & 0.974 \\
\hline & 26 & CC vs. AA & Random & 63.55 & $=0.001$ & 1.081 & $0.868-1.345$ & 0.694 & 0.488 & 0.427 & 0.401 \\
\hline & 26 & CA vs. AA & Random & 44.34 & 0.009 & 1.153 & $1.020-1.305$ & 2.268 & 0.023 & 0.860 & 0.569 \\
\hline & 26 & $C C+C A$ vs. $A A$ & Random & 89.63 & $=0.001$ & 1.085 & $0.828-1.422$ & 0.589 & 0.556 & 0.964 & 0.559 \\
\hline & 26 & $C C$ vs. $C A+A A$ & Random & 77.34 & $=0.001$ & 1.003 & $0.815-1.235$ & 0.030 & 0.976 & 0.171 & 0.254 \\
\hline \multicolumn{12}{|l|}{$\begin{array}{c}\text { By } \\
\text { Ethnicity }\end{array}$} \\
\hline \multirow[t]{5}{*}{ Caucasian } & 5 & C vs. A & Random & 67.19 & 0.016 & 0.992 & $0.797-1.235$ & -0.007 & 0.944 & 0.806 & 0.953 \\
\hline & 5 & CC vs. AA & Random & 65.33 & 0.021 & 0.959 & $0.572-1.608$ & -0.157 & 0.875 & 0.806 & 0.601 \\
\hline & 5 & CA vs. $A A$ & Random & 60.26 & 0.039 & 0.891 & $0.540-1.470$ & -0.452 & 0.651 & 1.000 & 0.869 \\
\hline & 5 & $\mathrm{CC}+\mathrm{CA}$ vs. $\mathrm{AA}$ & Random & 81.47 & $=0.001$ & 1.125 & $0.569-2.223$ & 0.339 & 0.735 & 0.462 & 0.252 \\
\hline & 5 & $C C$ vs. $C A+A A$ & Random & 55.56 & 0.061 & 1.071 & $0.922-1.245$ & 0.895 & 0.371 & 0.462 & 0.456 \\
\hline \multirow[t]{5}{*}{ Asian } & 17 & C vs. A & Random & 73.59 & 0.001 & 1.153 & $1.007-1.320$ & 2.057 & 0.040 & 0.224 & 0.664 \\
\hline & 17 & CC vs. AA & Random & 59.74 & 0.001 & 1.193 & $0.937-1.519$ & 1.429 & 0.153 & 0.029 & 0.003 \\
\hline & 17 & CA vs. AA & Random & 40.21 & 0.044 & 1.218 & $1.076-1.379$ & 3.111 & 0.002 & 0.536 & 0.356 \\
\hline & 17 & $C C+C A$ vs. $A A$ & Random & 92.39 & $=0.001$ & 1.133 & $0.810-1.585$ & 0.728 & 0.467 & 0.483 & 0.648 \\
\hline & 17 & $C C$ vs. $C A+A A$ & Random & 81.80 & $=0.001$ & 1.050 & $0.755-1.461$ & 0.290 & 0.771 & 0.052 & 0.013 \\
\hline \multirow[t]{5}{*}{ Latinos } & 5 & C vs. A & Random & 87.97 & $=0.001$ & 1.053 & $0.660-1.681$ & 0.216 & 0.829 & 0.806 & 0.759 \\
\hline & 5 & CC vs. AA & Random & 80.95 & 0.001 & 0.518 & $0.151-1.776$ & -1.047 & 0.295 & 0.308 & 0.373 \\
\hline & 5 & CA vs. $A A$ & Fixed & 20.76 & 0.286 & 1.001 & $0.707-1.418$ & 0.007 & 0.995 & 1.000 & 0.737 \\
\hline & 5 & $\mathrm{CC}+\mathrm{CA}$ vs. $\mathrm{AA}$ & Fixed & 55.11 & 0.083 & 0.925 & $0.667-1.283$ & -0.469 & 0.639 & 1.000 & 0.591 \\
\hline & 5 & $C C$ vs. $C A+A A$ & Random & 65.75 & 0.033 & 0.787 & $0.491-1.261$ & -0.997 & 0.319 & 0.734 & 0.757 \\
\hline \multicolumn{12}{|c|}{ High Quality Studies } \\
\hline & 20 & C vs. A & Random & 77.22 & $=0.001$ & 1.154 & $1.004-1.326$ & 2.012 & 0.044 & 0.417 & 0.791 \\
\hline & 20 & CC vs. AA & Random & 54.37 & 0.002 & 1.191 & $0.989-1.342$ & 1.820 & 0.069 & 0.381 & 0.717 \\
\hline & 20 & CA vs. AA & Random & 45.14 & 0.015 & 1.131 & $0.982-1.304$ & 1.710 & 0.087 & 0.721 & 0.873 \\
\hline & 20 & $\mathrm{CC}+\mathrm{CA}$ vs. $\mathrm{AA}$ & Random & 63.33 & $=0.001$ & 1.176 & $0.997-1.387$ & 1.930 & 0.054 & 0.256 & 0.630 \\
\hline & 20 & $C C$ vs. $C A+A A$ & Fixed & 31.81 & 0.086 & 1.079 & $0.961-1.211$ & 1.285 & 0.199 & 0.040 & 0.029 \\
\hline
\end{tabular}

TABLE 4 - The meta-analysis of IL-10 -819T>C polymorphism and risk of GC

\begin{tabular}{|c|c|c|c|c|c|c|c|c|c|c|c|}
\hline \multirow{2}{*}{ Subgroup } & \multirow{2}{*}{$\begin{array}{l}\text { Study } \\
\text { number }\end{array}$} & \multirow{2}{*}{ Genetic model } & \multirow{2}{*}{$\begin{array}{l}\text { Type of } \\
\text { model }\end{array}$} & \multicolumn{2}{|c|}{ Heterogeneity } & \multicolumn{4}{|c|}{ Odds ratio } & \multicolumn{2}{|c|}{ Publication Bias } \\
\hline & & & & $12(\%)$ & $\mathrm{PH}$ & OR & $95 \% \mathrm{Cl}$ & Ztest & POR & PBeggs & PEggers \\
\hline \multirow[t]{5}{*}{ Overall } & 18 & C vs. T & Random & 58.48 & 0.001 & 1.057 & $0.950-1.177$ & 1.017 & 0.309 & 0.820 & 0.381 \\
\hline & 18 & CC vs. TT & Random & 46.47 & 0.016 & 0.987 & $0.795-1.225$ & -0.120 & 0.905 & 0.544 & 0.469 \\
\hline & 18 & CT vs. TT & Random & 44.86 & 0.021 & 1.092 & $0.943-1.264$ & 1.171 & 0.242 & 0.324 & 0.376 \\
\hline & 18 & $\mathrm{CC}+\mathrm{CT}$ vs. TT & Random & 55.29 & 0.002 & 1.078 & $0.923-1.259$ & 0.948 & 0.343 & 0.404 & 0.621 \\
\hline & 18 & CC vs. $\mathrm{CT}+\mathrm{TT}$ & Fixed & 25.96 & 0.150 & 1.003 & $0.890-1.131$ & 0.056 & 0.955 & 0.448 & 0.492 \\
\hline \multicolumn{12}{|l|}{$\begin{array}{c}\text { By } \\
\text { Ethnicity }\end{array}$} \\
\hline \multirow[t]{5}{*}{ Caucasian } & 3 & C vs. T & Fixed & 50.64 & 0.132 & 1.086 & $0.914-1.289$ & 0.937 & 0.349 & 1.000 & 0.982 \\
\hline & 3 & CC vs. TT & Random & 66.66 & 0.050 & 1.008 & $0.474-2.144$ & 0.021 & 0.983 & 1.000 & 0.753 \\
\hline & 3 & CT vs. TT & Fixed & 59.86 & 0.083 & 0.803 & $0.524-1.232$ & -1.004 & 0.315 & 1.000 & 0.799 \\
\hline & 3 & $\mathrm{CC}+\mathrm{CT}$ vs. TT & Random & 67.42 & 0.046 & 0.938 & $0.445-1.980$ & -0.167 & 0.867 & 1.000 & 0.744 \\
\hline & 3 & CC vs. $C T+T T$ & Fixed & 0.00 & 0.552 & 1.163 & $0.941-1.438$ & 1.398 & 0.162 & 1.000 & 0.979 \\
\hline \multirow[t]{5}{*}{ Asian } & 14 & C vs. T & Random & 63.82 & 0.001 & 1.046 & $0.924-1.184$ & 0.708 & 0.479 & 0.742 & 0.499 \\
\hline & 14 & CC vs. TT & Random & 49.48 & 0.018 & 0.987 & $0.778-1.254$ & -0.104 & 0.917 & 0.661 & 0.545 \\
\hline & 14 & CT vs. TT & Random & 42.40 & 0.047 & 1.132 & $0.980-1.307$ & 1.684 & 0.092 & 0.742 & 0.879 \\
\hline & 14 & $\mathrm{CC}+\mathrm{CT}$ vs. TT & Random & 57.22 & 0.004 & 1.105 & $0.942-1.295$ & 1.224 & 0.221 & 0.584 & 0.826 \\
\hline & 14 & CC vs. CT+ TT & Fixed & 20.33 & 0.232 & 0.917 & $0.792-1.062$ & -1.157 & 0.247 & 0.125 & 0.170 \\
\hline \multicolumn{12}{|c|}{ High Quality Studies } \\
\hline & 15 & C vs. T & Random & 54.22 & 0.006 & 1.085 & $0.966-1.219$ & 1.377 & 0.169 & 0.552 & 0.391 \\
\hline & 15 & CC vs. TT & Random & 52.77 & 0.009 & 0.974 & $0.742-1.278$ & -0.191 & 0.848 & 0.620 & 0.488 \\
\hline & 15 & CT vs. TT & Random & 52.86 & 0.008 & 1.077 & $0.894-1.297$ & 0.779 & 0.436 & 0.276 & 0.326 \\
\hline & 15 & $\mathrm{CC}+\mathrm{CT}$ vs. TT & Random & 61.51 & 0.001 & 1.063 & $0.874-1.294$ & 0.611 & 0.541 & 0.198 & 0.460 \\
\hline & 15 & CC vs. CT+ TT & Fixed & 30.61 & 0.125 & 0.980 & $0.848-1.132$ & -0.275 & 0.784 & 0.322 & 0.150 \\
\hline
\end{tabular}



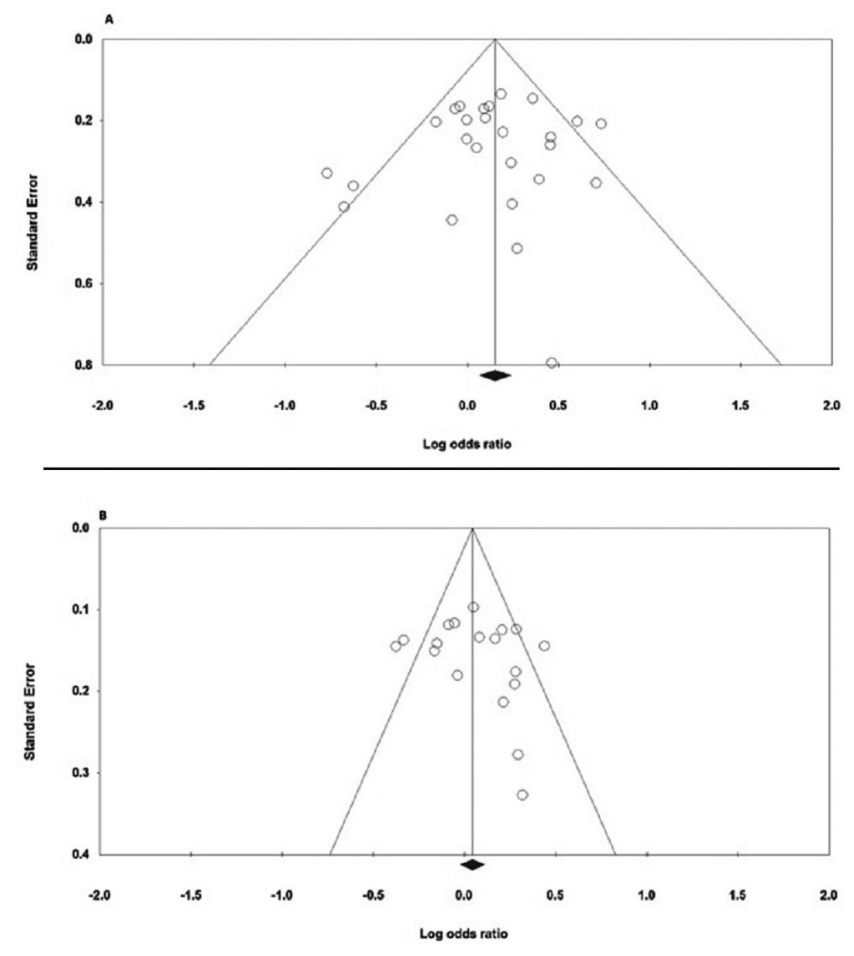

FIGURE 3 - Funnel plot for publication bias in the meta-analysis of the IL-10-592A >C and -819T>C Polymorphisms with GC: A) -592A>C (heterozygote model: CA vs. AA); B) -819T >C (allele model: C vs. T).

\section{DISCUSSION}

A meta-analysis can combine results from individual studies to overcome the limitation of small sample sizes and inadequate statistical power, produce a single estimate of the major effect, answer questions not resolved by the individual studies, resolve controversial debates arising from conflicting studies and cite limitations of current knowledge ${ }^{12,15}$. To date, several meta-analyses have been performed to evaluate the association of the IL-10 gene promoter $-592 \mathrm{~A}>\mathrm{C}$ and $-819 \mathrm{~T}>\mathrm{C}$ polymorphisms with GC. However, due to lack of ability to obtain overall reliable conclusions because of limited sample sizes, a consensus has not been reached. Therefore, to better elucidate the association of the IL-10 -592A >C and -819T >C polymorphisms with $\mathrm{GC}$, we performed an updated and more comprehensive meta-analysis by collecting 44 relevant casecontrol studies in 29 publications.

In 2014, Qi et al., in a meta-analysis of twelve studies, with 2,116 GC cases and 4,077 controls, reported that there was no significant association between the IL-10 -592C>A polymorphism and GC risk in overall population ${ }^{35}$. Since then, a series of better designed case-control studies on the association between IL-10 -592C > A polymorphism and GC were performed. Therefore, their results as a meta-analysis essentially remain an open field. In the current meta-analysis, 26 eligible case-control studies with 5,332 cases and 8,272 controls were identified and analyzed. Our results showed that there was a significant association between the IL-10 -592C $>A$ polymorphism and susceptibility to $\mathrm{GC}$ in total population. Moreover, compared with Qi et al meta-analysis the allele genetic model and subgroup analysis among Latinos were also carried out. In this meta-analysis we found that the IL-10 $-592 C>$ A polymorphism was associated with GC risk in Asians under the allele model ( $C$ vs. $A: O R=1.153,95 \% C l=1.007-1.320$, $\mathrm{p}=0.040$ ) and the heterozygote model (CA vs. $A A: O R=1.218$, $95 \% \mathrm{Cl}=1.076-1.379, \mathrm{p}=0.002)$. In addition, it is worth noting that the association between IL-10 $-592 \mathrm{C}>\mathrm{A}$ polymorphism and $\mathrm{GC}$ risk was significant by studies quality under the allele model $(\mathrm{OR}=1.154,95 \% \mathrm{Cl}=1.004-1.326, \mathrm{p}=0.044)$.

In 2016, Cui et al., performed a meta-analysis to assess the susceptibility of the IL-10 $-819 \mathrm{~T}>\mathrm{C}$ polymorphism to GC including eleven articles with 1,960 cases and 3,705 controls ${ }^{4}$. Their results suggested that L-10 $-819 \mathrm{~T}>\mathrm{C}$ polymorphism has a protective role in susceptibility to GC. Although their results suggested that the IL-10-819T >C polymorphism might not contribute to the risk of GC; however, these studies were with small number of cases and controls. In the current metaanalysis, we included a total of 18 case-control studies with 3,431 cases and 6,109 controls. The pooled results indicated that there was no obvious association between IL-10-819T >C polymorphism to GC. Therefore, our meta-analysis not only confirmed Cui et al results, but also provided most reliable statistical results by including more seven case-control studies ${ }^{4}$.

Heterogeneity between studies is common in the metaanalysis of genetic association studies ${ }^{41,49}$. In each case, the heterogeneity could be a result of different covariates such as ethnicity, sources of controls, sample size, HWE and methods used and so on ${ }^{16,17,30}$. In the current meta-analysis, significant between-study heterogeneity was detected across studies under all genetic models and thus we selected the randomeffects model to summarize the ORs. Therefore, we performed meta-regression analysis to find the source of between-study heterogeneity. The results showed that ethnicity and studies quality did not contribute to substantial between-study heterogeneity in the current meta-analysis. Moreover, we have performed sensitivity analysis according to sample size and leave-one-out analysis to determine whether modification of the inclusion criteria by removing one study each time affected the results. However, for both IL-10 -592A $>C$ and $-819 T>C$ polymorphisms, the sensitivity analyses did not materially affected the original results.

The present meta-analysis has some advantages compared to the previous meta-analyses. However, it does have some limitations that should be taken into account. First, we have included only studies published in the English and Chinese language in this meta-analysis; therefore, publication bias may have occurred. Second, in this meta-analysis the great proportion of statistical power was contributed by the Asian ethnicity. There were not enough studies in Caucasians and Latinos, which limited the statistical power. Moreover, African was one of the three largest ethnics, but we have not found any study on Africans. Third, the current meta-analysis was performed to analyze these polymorphisms separately; however, a haplotype analysis may have been more powerful for finding significant associations with GC. Forth, the ORs extracted from each eligible study were based on unadjusted estimates, while a more precise analysis should be performed in all individual data available, which would allow for the adjustment by other co-variants including age, environmental exposures, smoking status, and other lifestyle factors. Finally, gene-gene and gene-environment interactions which may modulate the GC susceptibility were not addressed in this meta-analysis for the lack of sufficient data.

\section{CONCLUSION}

The current meta-analysis results inconsistent with the previous meta-analyses showed that the IL-10 -592A $>C$ polymorphism contributed to the susceptibility of GC in overall population, particularly in Asian populations. However, the IL-10 -819T> C polymorphism was not associated with an increased risk of GC. Further large well-designed studies are still needed to determine the effects of the IL-10 -592A $>C$ and $-819 T>C$ polymorphisms on GC. 
REFERENCES

1. Alpízar-Alpízar W, Pérez-Pérez GI, Une C, Cuenca P, Sierra R. Association ofinterleukin-1Bandinterleukin-1RN polymorphismswith gastric cance in a high-risk population of Costa Rica. Clin Exp Med. 2005;5(4):169-176 doi:10.1007/s10238-005-0082-3.

2. Begg CB, Mazumdar M. Operating characteristics of a rank correlation test for publication bias. Biometrics. 1994;50(4):1088-1101.

3. Con SA, Takeuchi H, Con-Chin GR, Con-Chin VG, Yasuda N, Con-Wong R. Role of bacterial and genetic factors in gastric cancer in Costa Rica. World J Gastroenterol. 2009;15(2):211-218. doi:10.3748/WJG.15.211.

4. Cui X, Huang Q, Li X, Liu F, Wang D, Yan D, et al. Relationship between Interleukin-10 Gene C-819T Polymorphism and Gastric Cancer Risk: Insights from a Meta-Analysis. Med Sci Monit. 2016;22:2839-2845

5. Crusius JB1, Canzian F, Capellá G, Peña AS, Pera G, Sala N, et al. Cytokine gene polymorphisms and the risk of adenocarcinoma of the stomach in the European prospective investigation into cancer and nutrition (EPIC-EURGAST). Ann Oncol. 2008;19(11):1894-1902. doi:10.1093/ annonc/mdn400.

6. de Oliveira JG, Rossi AF, Nizato DM, Cadamuro AC, Jorge YC, Valsech $\mathrm{MC}$, et al. Influence of functional polymorphisms in TNF-a, IL-8, and IL-10 cytokine genes on mRNA expression levels and risk of gastric cancer Tumor Biol. 2015;36(12):9159-9170. doi:10.1007/s13277-015-3593-x.

7. DerSimonian R, Laird N. Meta-analysis in clinical trials. Control Clin Trials. 1986;7(3):177-188.

8. DengW,HeL, PengX. [TherelationshipamongIL-10genepolymorphisms $\mathrm{H}$ elicobacter pylori and noncardia gastric cancer development in Fujian province]. [Article in Chinese]. Wei Chang Bing Xue He Gan Bing Xue Za Zhi. 2008:17:212-214.

9. Egger M, Davey Smith G, Schneider M, Minder C. Bias in meta-analysis detected by a simple, graphical test. BMJ. 1997;315(7109):629-634.

10. El-OmarEM, Rabkin CS, GammonMD, VaughanTL, RischHA, Schoenberg $J B$, et al. Increased risk of noncardia gastric cancer associated with proinflammatory cytokine gene polymorphisms. Gastroenterology. 2003;124(5):1193-1201

11. García-GonzálezMA, LanasA, QuinteroE, NicolásD, Parra-BlancoA, Strunk M, Benito R, et al. Gastric Cancer Susceptibility Is Not Linked to Pro-and Anti-InflammatoryCytokineGenePolymorphismsinWhites:ANationwide Multicenter Study in Spain. Am J Gastroenterol. 2007;102(9):1878-1892. doi:10.1111/j.1572-0241.2007.01423.x

12. Garg AX, HackamD, TonelliM.SystematicReviewand Meta-analysis:When One Study Is Just not Enough. Clin J Am Soc Nephrol. 2008;3(1):253-260. doi:10.2215/CJN.01430307.

13. Gonzalez-Hormazabal P, Musleh M, Bustamante M, StambukJ, Escandar $\mathrm{S}$, Valladares $\mathrm{H}$, et al. Role of cytokine gene polymorphisms in gastric cancer risk in Chile. Anticancer Res. 2014;34(7):3523-3530.

14. He B, Pan Y, Xu Y, Nie Z, Chen L, Gu L, et al. Increased Risk for Gastric Cancer in Carriers of the Lymphotoxin-a+252G Variant Infected by Helicobacter pylori. Genet Test Mol Biomarkers. 2012;16(1):9-14 doi:10.1089/gtmb.2011.0078.

15. Haidich AB, Haidich AB. Meta-analysis in medical research. Hippokratia 2010;14(Suppl 1):29-37.

16. Jafari Nedooshan J, Kargar $\mathrm{S}$, Neamatzadeh $\mathrm{H}$, Haghighi F, Dehghan Mohammad-Abadi R, Seddighi N. Lack of Association of the Fat Mass and Obesity Associated (FTO) Gene rs9939609 Polymorphism with Breast Cancer Risk: a Systematic Review and Meta-Analysis Based on Case - Control Studies. Asian Pac J Cancer Prev. 2017;18(4):1031-1037. doi:10.22034/APJCP.2017.18.4.1031.

17. Jafari Nedooshan J, Forat-Yazdi M, Neamatzadeh $H$, Zare Shehneh $M$ Kargar S, Seddighi N. Genetic Association of XRCC1 Gene rs1799782 rs25487 and rs25489 Polymorphisms with Risk of Thyroid Cancer: MetaAnalysis. Asian Pac J Cancer Prev. 2017;18(1):263-270.

18. Kamali M, Hantoushzadeh S, Borna S, Neamatzadeh H, Mazaheri M Noori-Shadkam M, et al. Association between Thrombophilic Genes Polymorphisms and Recurrent Pregnancy Loss Susceptibility in the Iranian Population: a Systematic Review and Meta-Analysis. Iran Biomed J. 2018:22(2):78-89.

19. Kamangar F, Abnet CC, Hutchinson AA, Newschaffer CJ, Helzlsouer K Shugart $Y Y$, et al. Polymorphisms in inflammation-related genes and risk of gastric cancer (Finland). Cancer Causes Control. 2006;17(1):117-125. doi:10.1007/s10552-005-0439-7.

20. Kang JM, Kim N, Lee DH, Park JH, Lee MK, Kim JS, et al. The effects of genetic polymorphisms of IL-6, IL-8, and IL-10 on Helicobacter pylori-induced gastroduodenal diseases in Korea. J Clin Gastroenterol. 2009:43(5):420-428. doi:10.1097/MCG.0b013e318178d1d3.

21. Khoram-Abadi KM, Forat-Yazdi M, Kheirandish S, Saeidi N, Zarezade Z, Mehrabi N, et al. DNMT3B -149 C>T and -579 G>T polymorphisms and risk of gastric and colorectal cancer: A meta-analysis. Asian Pacific J Cancer Prev. 2016;17(6)

22. Kim J, Cho YA, Choi IJ, LeeYS, Kim SY, Shin A, et al. Effects of interleukin-10 polymorphisms, Helicobacter pylori infection, and smoking on the risk of noncardia gastric cancer. Katoh M, ed. PLoS One. 2012;7(1):e29643 doi:10.1371/journal.pone.0029643.
23. Kumar S, Kumari N, Mittal RD, Mohindra S, Ghoshal UC. Association between pro-(IL-8) and anti-inflammatory (IL-10) cytokine variants and their serum levels and $\mathrm{H}$. pylori-related gastric carcinogenesis in northern India. Meta Gene. 2015;6:9-16. doi:10.1016/j.mgene.2015.07.008

24. Kuo WH, Huang CY, Fu CK, Hsieh YH, Liao CH, Hsu CM,et al. Effects of interleukin-10 polymorphisms and smoking on the risk of gastric cancer in Taiwan. In Vivo. 2014;28(5):967-971.

25. Lee JY, Kim HY, Kim KH, Kim SM, Jang MK, Park JY, et al. Association of polymorphism of IL-10 and TNF-A genes with gastric cancer in Korea. Cancer Lett. 2005;225(2):207-214. doi:10.1016/j.canlet.2004.11.028.

26. Li L, Tang XY, Ye LM, Yang Q, LiY. Investigation on the association between IL-10 C819T gene polymorphisms and susceptibility to gastric cancer. Genet Mol Res. 2016;15(4). doi:10.4238/gmr15047915.

27. Liu J, Song B, Wang J-L, Li Z-J, Li W-H, Wang Z-H. Polymorphisms of interleukin-10 promoter are not associated with prognosis of advanced gastric cancer. World J Gastroenterol. 2011;17(10):1362. doi:10.3748/ wjg.v17.i10.1362.

28. Ma B, Zhang T, Wan Y, Zhan F. [A case-control study of interleukin-10 gene $-592 \mathrm{~A} / \mathrm{C}$ polymorphism and the risk of gastric cancer]. [Article in Chinese].

29. Mantel N, Haenszel W. Statistical aspects of the analysis of data from retrospective studies of disease. J Natl Cancer Inst. 1959;22(4):719-748.

30. Mehdinejad M, Sobhan MR, Mazaheri M, Shehneh MZ, Neamatzadeh $\mathrm{H}$, Kalantar SM. Genetic association between ERCC2, NBN, RAD51 gene variants and osteosarcoma risk: A systematic review and meta-analysis. AsianPacificJCancerPrev.2017;18(5).doi:10.22034/APJCP.2017.18.5.1315.

31. Mocellin S, Marincola FM, Young HA. Interleukin-10 and the immune response against cancer: a counterpoint. J Leukoc Biol. 2005;78(5):10431051. doi:10.1189/jlb.0705358.

32. Namazi A, Forat-Yazdi $M$, Jafari $M$, Farahnak $S$, Nasiri $R$, Foroughi $E$, et al. Association of Interleukin-10 -1082 a/G (Rs1800896) Polymorphism With Susceptibility To Gastric Cancer: Meta-Analysis of 6,101 Cases and 8,557 Controls. Arq Gastroenterol. 2018:55(1):33-40. doi:10.1590/ s0004-2803.201800000-18.

33. Oh SS, Chang SC, Cai L, Cordon-Cardo C, Ding BG, Greenland S, et al. Single nucleotide polymorphisms of 8 inflammation-related genes and their associations with smoking-related cancers. Int J cancer. 2010;127(9):2169-2182. doi:10.1002/ijc.25214.

34. Pan XF, Yang SJ, Loh $M$, Xie Y, Wen YY, Tian Z, et al. Interleukin-10 gene promoter polymorphisms and risk of gastric cancer in a Chinese population: single nucleotide and haplotype analyses. Asian PacJ Cancer Prev. 2013;14(4):2577-2582.

35. QiM,LiuDM,PanLL, LinYX. Interleukin-10gene-592C\&gt;A polymorphism and susceptibility to gastric cancer.GenetMolRes.2014;13(4):8954-8961. doi:10.4238/2014.October.31.10

36. Sahami-Fard MH, Yazd EF, KhazaeiZ, Neamatzadeh H.Lack ofassociation between the $\mathrm{CDH} 1-160 \mathrm{CC}>$ A polymorphismand risk of gastrointestinal cancer-Ameta-analysis.AsianPacificJCancerPrev.2016;17(5).doi:10.7314/ APJCP.2016.17.5.2415

37. Savage SA, Abnet CC, Haque K, Mark SD, Qiao YL, Dong ZW, et al. Polymorphisms in interleukin $-2,-6$, and -10 are not associated with gastric cardia or esophageal cancer in a high-risk Chinese population. Cancer Epidemiol Biomarkers Prev. 2004;13(9):1547-1549.

38. Shi J, Qu Y-P, Hou P. Pathogenetic mechanisms in gastric cancer. World J Gastroenterol. 2014;20(38):13804. doi:10.3748/wjg.v20.i38.13804.

39. Sicinschi LA, Lopez-Carrillo L, Camargo MC, Correa P, Sierra RA, Henry $\mathrm{RR}$, et al. Gastric cancer risk in a Mexican population: role of Helicobacter pylori CagA positive infection and polymorphisms in interleukin-1 and -10 genes. Int J cancer. 2006;118(3):649-657. doi:10.1002/ijc.21364.

40. Siegel RL, Miller KD, Jemal A. Cancer statistics, 2016. CA Cancer J Clin. 2016;66(1):7-30. doi:10.3322/caac.21332

41. Sobhan MR, MehdinejadM,JamaladiniMH,MazaheriM,Zare-ShehnehM, NeamatzadehH.Association betweenasparticacidrepeatpolymorphism of the asporin gene and risk of knee osteoarthritis: A systematic review and meta-analysis.ActaOrthop TraumatolTurc.2017;51(5).doi:10.1016/j. aott.2017.08.001.

42. Su SP, Yang ZB, Tian YL, Xiang Y, Wu M, Ma X. Relationship between Polymorphisms of IL-1ß-31, IL-10-819 and TNF-a-1031 Genes and Susceptibilities to H. Pylori Infection-Associated Gastric Ulcerand Cancer. Chinese Journal of Biologicals. 2010;23(5):517-520.

43. Sugimoto $M$, Furuta $T$, Shirai $N$, Nakamura $A$, Kajimura M, Sugimura $H_{\text {, }}$ et al. Effects of interleukin-10 gene polymorphism on the development of gastric cancer and peptic ulcer in Japanese subjects. J Gastroentero Hepatol. 2007;22(9):1443-1449. doi:10.1111/j.1440-1746.2006.04613.x

44. Tan P, Yeoh K-G. Genetics and Molecular Pathogenesis of Gastric Adenocarcinoma.Gastroenterology.2015;149(5):1153-1162.e3.doi:10.1053/J. gastro.2015.05.059.

45. Wang $\mathrm{P}$, An J, Zhu Y, Wan X, Zhang $\mathrm{H}$, Xi S, et al. Association of three promoterpolymorphismsininterleukin-10genewithcancersusceptibility intheChinese population:ameta-analysis. Oncotarget.2017;8(37):6238262399. doi:10.18632/oncotarget.18220.

46. Wu MS, Wu CY, Chen CJ, Lin MT, Shun CT, Lin JT. Interleukin-10 genotypes associate with the risk of gastric carcinoma in Taiwanese Chinese. Int J cancer. 2003;104(5):617-623. doi:10.1002/ijc.10987. 
47. Xiao $H$, Jiang $Y$, Li R, Xia B. [Association of IL-10 gene polymorphisms with gastroduodenal diseases in Hubei Han population]. Zhonghua Yi Xue Yi Chuan Xue Za Zhi. 2009;26(4):423-426.

48. Xue H, Lin B, An J, Zhu Y, Huang G. Interleukin-10-819 promoter polymorphism in association with gastric cancer risk. BMC Cancer. 2012;12(1):102. doi:10.1186/1471-2407-12-102.

49. Yazdi MM, Jamalaldini MH, Sobhan MR, Jafari M, Mazaheri M, ZareShehneh M, etal. Association of ESR $\alpha$ Gene PvullT $>C, X b a l A>G$ and Btgl G>APolymorphismswith KneeOsteoarthritisSusceptibility:ASystematic Review and Meta-Analysis Based on 22 Case-Control Studies. Arch Bone Jt Surg. 2017;5(6). doi:10.22038/abjs.2017.22391.1578.

50. Yin J, Wang X, WeiJ, Wang L, ShiY, Zheng L, etal. Interleukin $12 B$ rs3212227 $T>G$ polymorphism was associated with an increased risk of gastric cardiac adenocarcinoma in a Chinese population. Dis esophagus Off J Int Soc Dis Esophagus. 2015;28(3):291-298. doi:10.1111/dote.12189.

51. Yuan LJ, Jin TB, Yin JK, Du XL, Wang Q, Dong R, et al. Polymorphisms of tumor-related genes IL-10, PSCA, MTRR and NOC3L are associated with the risk of gastric cancer in the Chinese Han population. Cancer Epidemiol. 2012;36(6):e366-e372. doi:10.1016/j.canep.2012.05.016.
52. Zabaleta J. Multifactorial Etiology of Gastric Cancer. Methods Mol Biol. 2012;863:411-35. doi: 10.1007/978-1-61779-612-8_26.

53. Zambon CF, Basso D, Navaglia F, Belluco C, Falda A, FogarP, etal. Pro- and anti-inflammatorycytokinesgenepolymorphismsand Helicobacterpylori infection: interactions influence outcome. Cytokine. 2005;29(4):141-152. doi:10.1016/j.cyto.2004.10.013.

54. Zeng X, Li Y, Liu T, Zhang J. Diverse H. pylori strains, IL-10 promoter polymorphisms with high morbidity of gastric cancer in Hexi area of Gansu Province, China. Mol Cell Biochem. 2012;362(1-2):241-248. doi:10.1007/s11010-011-1149-y.

55. Zhuang W, Wu XT, Zhou Y, Liu L, Liu GJ, Wu TX, et al. Interleukin10 -592 promoter polymorphism associated with gastric cancer among Asians: a meta-analysis of epidemiologic studies. Dig Dis Sci. 2010;55(6):15251532. doi:10.1007/s10620-009-0922-1. 